\title{
Vibration energy harvesting: from micro to macro scale
}

\author{
Wen-Ming Zhang ${ }^{1} \cdot$ Lei Zuo $^{2}$
}

Published online: 1 July 2020

(c) The Chinese Society of Theoretical and Applied Mechanics and Springer-Verlag GmbH Germany, part of Springer Nature 2020

Ambient vibrational energy is one kind of sources that has received growing research interests over the past two decades in energy harvesting at both small and large energy scales. With the advancement of microelectronics for sensing, computation and communication, it is possible for energy harvesting to enable self-powered wireless sensor networks, micro-electronic devices, wearable/portable electronics, Internet of Things in automotive, industrial, environmental, defense, aeronautics, civil structural, and medical applications. Besides the micro- and milli-watts energy, large-scale energy harvesting from oceans is promising to generate hundred watts or hundred thousand watts of electricity for solving the global energy crisis or powering the blue economy. The efficient design of the micro and macro energy harvesting systems require the understanding of the basic mechanics, mechatronics and electrodynamics of the scenario excitation, microelectromechanical system (MEMS) and macro vibration structures. Significant progress in vibration energy harvesting related to nonlinear dynamics has been made to increase the frequency bandwidth. Addressing the concerns from theoretical to experimental studies for high efficient vibration energy harvesting remains challenging.

This thematic issue consists of eight articles contributed by experts who are active on related topics. Yao et al. [1], Tan et al. [2] and Wang et al. [3] presented three studies of multiple-stable energy harvesting with magnetic interactions, reflecting the state-of-the-art developments on nonlinear dynamics applied in broadband vibration energy harvesting. Zhang and Wang [4] carries out parameter identification

Wen-Ming Zhang

wenmingz@sjtu.edu.cn

Lei Zuo

leizuo@vt.edu

1 State Key Laboratory of Mechanical System and Vibration, School of Mechanical Engineering, Shanghai Jiao Tong University, Shanghai 200240, China

2 Department of Mechanical Engineering, Virginia Tech, Blacksburg, VA 24061, USA for a nonlinear energy harvester via a dynamic frequency approach. Jiang et al. [5] investigate the energy harvesting by internal resonance in a spring-pendulum system. Qian et al. [6] improves the off-resonance energy harvesting performance using dynamic magnetic preloading. Meng et al. [7] perform modeling and experiments on Galfenol energy harvester. Liu et al. [8] give a comprehensive survey on the mechanisms of power take-off devices of ocean wave energy converters. We hope that these studies will bring new interests and inspirations to the field of vibration energy harvesting.

Finally, we appreciate the efforts made by all the authors for this special issue. We are grateful to the Associate Editor-in-Chief of Acta Mechanica Sinica, Prof. Liqun Chen, for inviting us as the guest editors of this issue on energy harvesting. We also thank Dr. Ting Tan of Shanghai Jiao Tong University and the staff of the editorial office of Acta Mechanica Sinica for managing, assistance, and support.

\section{References}

1. Yao, M., Liu, P., Ma, L., et al.: Experimental study on broadband bistable energy harvester with L-shaped piezoelectric cantilever beam. Acta Mech. Sin. 36(3), 557-577 (2020). https://doi. org/10.1007/s10409-020-00956-1

2. Tan, T., Yan, Z., Ma, K., et al.: Nonlinear characterization and performance optimization for broadband bistable energy harvester. Acta Mech. Sin. 36(3), 578-591 (2020). https://doi.org/10.1007/ s10409-020-00946-3

3. Wang, J., Geng, L., Zhou, S., et al.: Design, modeling and experiments of broadband tristable galloping piezoelectric energy harvester. Acta Mech. Sin. 36(3), 592-605 (2020). https://doi. org/10.1007/s10409-020-00928-5

4. Zhang, Z., Wang, W, Wang, C.: Parameter identification of nonlinear system via a dynamic frequency approach and its energy harvester application. Acta Mech. Sin. 36(3), 606-618 (2020). https://doi.org/10.1007/s10409-020-00972-1

5. Jiang, W., Han, X., Chen, L., et al.: Improving energy harvesting by internal resonance in a spring-pendulum system. Acta Mech. Sin. 36(3), 619-624 (2020). https://doi.org/10.1007/s10409-02000945-4 
6. Qian, F., Zhou, S., Zuo, L.: Improving the off-resonance energy harvesting performance using dynamic magnetic preloading. Acta Mech. Sin. 36(3), 625-635 (2020). https://doi.org/10.1007/s1040 9-020-00929-4

7. Meng, A., Yan, C., Li, M., et al.: Modeling and experiments on Galfenol energy harvester. Acta Mech. Sin. 36(3), 636-644 (2020). https://doi.org/10.1007/s10409-020-00943-6

8. Liu, Z., Zhang, R., Xiao, H., et al.: Survey of the mechanisms of power take-off (PTO) devices of wave energy converters. Acta Mech. Sin. 36(3), 645-660 (2020). https://doi.org/10.1007/s1040 9-020-00958-Z

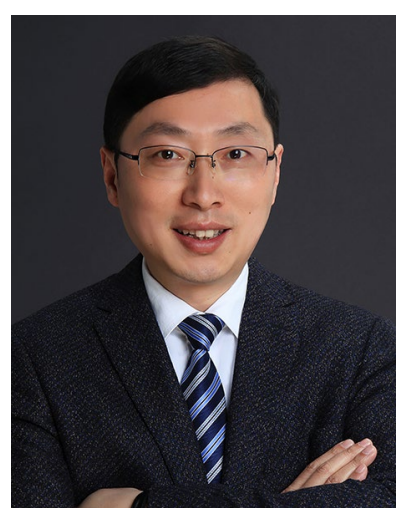

Wen-Ming Zhang received his Ph.D. degree in 2006 at School of Mechanical Engineering from Shanghai Jiao Tong University, China. Currently, he is a professor at School of Mechanical Engineering, Shanghai Jiao Tong University. His research focus is on dynamics and control in microelectromechanical system/ nanoelectromechanical system (MEMS/NEMS), soft robotics, nonlinear dynamics and vibration energy harvesting. Prof.
Zhang received the National Natural Science Fund for Distinguished Young Scientists of China in 2016. He is a member of the Chinese Society of Theoretical and Applied Mechanics (CSTAM), the American Society of Mechanical Engineers (ASME), and the Institute of Electrical and Electronics Engineers (IEEE).

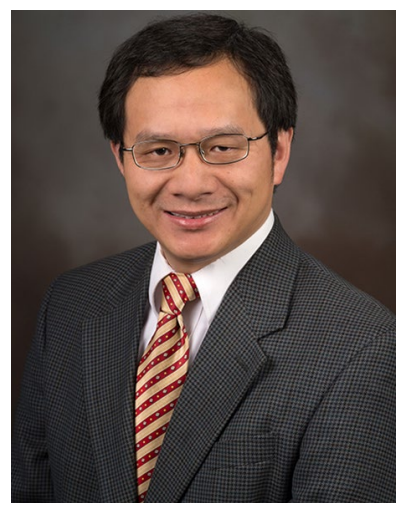

Lei Zuo completed his BS from Tsinghua University (1997) and $\mathrm{Ph} . \mathrm{D}$. from Massachusetts Institute of Technology (MIT) (2005). He is currently the Robert E. Hord Jr. Professor at Virginia Tech and the Director of the NSF I/UCRC Center for Energy Harvesting Materials and Systems. His research interests are design, dynamics, control and manufacturing of energy systems, with applications to ocean renewable energy, vehicles and transportation, smart infrastructures, self-powered sensors.

He was the sole recipient of the 2017 ASME Leonardo Da Vinci Award and the 2015 ASME Thar Energy Design Award and was selected for two R\&D 100 Awards. He has published nearly 300 papers, and advised $15 \mathrm{Ph}$. D. and over $40 \mathrm{MS}$ students to completion. Zuo is a Fellow of ASME. 